\title{
High Incidence of Hippocampal Abnormalities in Pediatric Patients with Congenital Cytomegalovirus Infection
}

\author{
Takenori Natsume ${ }^{1}$ Yuji Inaba ${ }^{1,2,3}$ Yoshihiro Osawa ${ }^{1}$ Tetsuhiro Fukuyama ${ }^{1}$ (1) \\ ${ }^{1}$ Department of Pediatrics, Shinshu University School of Medicine, \\ Matsumoto, Japan \\ 2 Division of Neuropediatrics, Nagano Children's Hospital, Azumino, \\ Japan \\ ${ }^{3}$ Life Science Research Center, Nagano Children's Hospital, Azumino, \\ Japan

\begin{abstract}
Address for correspondence Yuji Inaba, MD, PhD, Division of Neuropediatrics, Nagano Children's Hospital, 3100 Toyoshina, Azumino 399-8288, Japan (e-mail: inabay@shinshu-u.ac.jp).

Tetsuhiro Fukuyama, MD, PhD, Department of Pediatrics, Shinshu University School of Medicine, 3-1-1 Asahi, Matsumoto 390-8621, Japan (e-mail: fukuyama@shinshu-u.ac.jp).
\end{abstract}

Neuropediatrics 2022;53:239-245.

\author{
Abstract \\ Keywords \\ - brain magnetic \\ resonance imaging \\ - congenital \\ cytomegalovirus \\ infection \\ - hippocampal \\ malrotation \\ - hippocampus
}

Background Congenital cytomegalovirus (CMV) infection exhibits polymicrogyria, intracranial calcification, white matter lesions, and several types of intracranial lesions on magnetic resonance imaging (MRI), in addition to various developmental disorders and epilepsies. However, little is known on the presence of hippocampal abnormality in this affliction. The aim of this study is to clarify the incidence of hippocampal abnormality in congenital CMV infection.

Methods Seventeen children diagnosed as having congenital CMV infection along with 17 age-matched pediatric controls were retrospectively evaluated by brain MRI and clinical review. The measurement data were obtained from conventional coronal sections in this retrospective study. Hippocampal malrotation (HIMAL) was defined as a hippocampal diameter ratio (i.e., the ratio of the height and width of the hippocampus) of $>0.92$.

Results Hippocampal diameter ratios were significantly higher in the congenital CMV infection group ( 0.99 [range: $0.70-1.58$ ] on the right side and 0.85 [range: $0.66-1.39$ ] on the left side) than in controls ( 0.71 [range: $0.58-0.91$ ] and 0.70 [range: $0.50-1.00$ ], respectively). HIMAL was present in 17 of 34 hippocampi (50\%) in the congenital CMV infection group and 1 of 34 hippocampi (2.9\%) in controls. No correlations were detected between HIMAL and intelligence quotient/developmental quotient or the occurrences of autism spectrum disorder or epilepsy.

Conclusion This study is the first to demonstrate the incidence of hippocampal abnormality to be significantly higher in congenital CMV infection patients than in agematched controls. Further study is necessary to clarify the associations of HIMAL with other clinical and developmental features. received

September 11, 2021 accepted after revision January 24, 2022 published online April 12, 2022
DOI https://doi.org/ 10.1055/a-1754-1142. ISSN 0174-304X. (c) 2022. The Author(s).

This is an open access article published by Thieme under the terms of the Creative Commons Attribution-NonDerivative-NonCommercial-License, permitting copying and reproduction so long as the original work is given appropriate credit. Contents may not be used for commercial purposes, or adapted, remixed, transformed or built upon. (https://creativecommons.org/ licenses/by-nc-nd/4.0/)

Georg Thieme Verlag KG, Rüdigerstraße 14, 70469 Stuttgart, Germany 


\section{Introduction}

Congenital cytomegalovirus (CMV) infection is the most common congenital viral infection. CMV afflicts 0.5 to $2.4 \%$ of live births, ${ }^{1-3}$ although only 10 to $15 \%$ of infected infants display neurological symptoms. It is well known that congenital CMV infection can cause such neurological complications as intellectual disability, ${ }^{4}$ neurodevelopmental disorders, sensorineuronal hearing loss, ${ }^{5}$ and epilepsy. ${ }^{6}$ In addition, several neuroimaging abnormalities have been identified in CMV infection, including cerebral calcification, ventriculomegaly, migration abnormalities, cortical dysplasia, cortical atrophy, and cerebellar hypoplasia. ${ }^{7}$ Recent studies have also shown more trivial brain involvement; cerebral white matter lesions were detected by magnetic resonance imaging (MRI) in patients with congenital CMV infection not exhibiting obvious neurological symptoms. ${ }^{6,8}$ However, little is known on the presence of hippocampal abnormality in congenital CMV infection.

Hippocampal malrotation (HIMAL) is described as the atypical appearance of the hippocampus with abnormal medial location along the choroid fissure, round or pyramidal shape, and/or verticalization of the collateral sulcus with a blurred inner structure, and enlargement of the inferior horn of the lateral ventricle. ${ }^{9}$ Since it resembles the fetal orientation, HIMAL is presumed as the consequence of incomplete infolding of medial temporal lobe structures during embryonic brain development. ${ }^{9-11}$ A variety of criteria have been used in different studies on HIMAL. ${ }^{911-14}$ Tsai et al investigated the quantitative futures of HIMAL and reported hippocampal shape change with a hippocampal diameter ratio of $>0.8$, as key features in HIMAL diagnosis according to coronal sections were obtained by T1-weighted whole-brain structural 3-Tesla (T) MRI scans, using the protocol for epilepsy with oblique coronal imaging perpendicular to hippocampal long axis. ${ }^{15}$ This method is useful to quantify and diagnose HIMAL, even in children.
Our recent investigation on congenital CMV infection revealed significant correlations between intellectual quotient and cerebral white matter lesions, ${ }^{8}$ with a higher incidence of enlargement of the inferior horn of the lateral ventricle (unpublished data) which led us to speculate that congenital CMV infection could affect the shape of hippocampus. In addition, regardless of the presence or absence of cortical dysplasia, neurodevelopmental disorders are common in patients with congenital CMV infection, and we hypothesized that hippocampal changes could be a causative lesion in neurodevelopmental disorders. However, no reports have addressed the association between congenital CMV infection and hippocampus shape to date. The present study examined the incidence of HIMAL using the quantitative index of brain MRI in patients with congenital CMV infection.

\section{Materials and Methods}

We retrospectively evaluated the brain MRI results from 17 pediatric patients diagnosed as having congenital CMV infection between 2011 and 2017, as well as 17 age-matched children as controls ( - Table $\mathbf{1}$ ). The diagnosis of congenital CMV infection was based on the retrospective analysis of preserved dried umbilical cords according to the method described by Furutate et al. ${ }^{16}$ Briefly, DNA was extracted from umbilical cords, and CMV DNA was detected using the quantitative polymerase chain reaction. Patients having hematological diseases without central nervous system involvement who underwent brain MRI before treatment were employed as the control participants.

Brain MRI was performed after the age of 12 months in all patients using a 1.5-T scanner. T2-weighted images were used according to the routine protocol for obtaining coronal sections perpendicular to the anterior commissure-posterior commissure (AC-PC) line. MRI was conducted under the following conditions: repetition time $=3,000$ to $6,500 \mathrm{~ms}$,

Table 1 Characteristics of the cCMV group and control group

\begin{tabular}{|l|l|l|l|}
\hline & cCMV group & Control group & $p$-Value \\
\hline Number & 17 & 17 & \\
\hline Age (y) & $2.94 \pm 1.85$ & $3.06 \pm 1.30$ & NS \\
\hline Gender (F/M) & $8 / 9$ & $8 / 9$ & NS \\
\hline ASD & 8 & 0 & 0.001 \\
\hline IQ & $75.8 \pm 26.1$ & NA & \\
\hline Deafness & 15 & 0 & $<0.001$ \\
\hline MRI findings & & & \\
\hline Cortical dysplasia & 2 & 0 & NS \\
\hline Cerebral atrophy & 2 & 0 & NS \\
\hline Hippocampal sclerosis & 0 & 0 & NS \\
\hline Hippocampal atrophy & 1 & 0 & NS \\
\hline White matter lesion & 14 & 0 & $<0.001$ \\
\hline
\end{tabular}

Abbreviations: ASD, autism spectrum disorder; cCMV, congenital cytomegalovirus infection; F, female; IQ, intelligence quotient; M, male; MRI, magnetic resonance imaging; NA, not available; NS, not significant. 
echo time $=78$ to $120 \mathrm{~ms}$, and slice thickness $=3$ to $5 \mathrm{~mm}$. The hippocampal diameter $(=\mathrm{d} / \mathrm{e}$, where $\mathrm{d}=$ the height of the hippocampus, and $\mathrm{e}=$ the width of the hippocampus) was calculated on the slice where the width of hippocampus was maximal in the hippocampal body (-Fig. 1A). Mean hippocampal angle was earlier reported as 29-degrees (range: 17-42 degrees) caudal to the AC-PC line in healthy elderly volunteers. ${ }^{17}$ If the hippocampal angle was assumed to be 30 degrees, the hippocampal height in coronal crosssectional images perpendicular to the AC-PC line would be $2 /$ $\sqrt{3}$ times higher than that to the hippocampal long axis. Accordingly, the cut-off value of the hippocampal diameter ratio was set as 0.92 (i.e., $0.8 \times 2 / \sqrt{3}$ ) based on the postulate of Tsai et al. ${ }^{15}$ Similarly, the medial distance ratio $(=\mathrm{f} / \mathrm{g}$, where $\mathrm{f}=$ the distance from the midline to the medial hippocampus, and $\mathrm{g}=$ the distance from the midline to the temporal neocortex) was calculated (-Fig. 1B). One of the cases of HIMAL also showed enlargement of the inferior horn of the lateral ventricle (-Fig. 1C). All measurements were performed separately by three pediatric neurologists who were blinded to the clinical findings, and final values were calculated as the mean score for each parameter. To evaluate interrater reliability, we calculated the intraclass correlation coefficients from the measured data for the hippocampal diameter ratio and medial distance ratio.

We also evaluated MRI findings for cortical dysplasia, cerebral atrophy, hippocampal sclerosis (hippocampal atrophy and T2 high signal), hippocampal atrophy, and white matter lesion (- Table $\mathbf{1}$ ).

The patients with congenital CMV infection were tested for hearing function, intelligence quotient (IQ) using the Wechsler intelligence scale for children, fourth edition, for 5 years of age and older, developmental quotient (DQ) using the Kyoto Scale of Psychological Development 2001 for under 5 years of age, and autism spectrum disorder based on the Diagnostic and Statistical Manual of Mental Disorder, fifth edition.

Welch's $t$-test was adopted to evaluate the significance of differences in hippocampal diameter ratio in both groups. Differences in clinical characteristics and the occurrence rate of HIMAL between the groups were compared using the Chisquare test. Relationships of hippocampal diameter ratio and IQ or DQ were analyzed by Pearson's correlation coefficient test. The level of significance was set as a $p$-value of $<0.05$. Statistical analyses were conducted using SPSS version 26 software (IBM Corp, Armonk, New York, United States).

This study was approved by the ethics committee of Shinshu University School of Medicine (identifier no. 4021). Written informed consent was obtained from all parents or guardians.

\section{Results}

The clinical characteristics of the 17 patients in the congenital CMV infection group and the 17 control participants are summarized in - Table 1. There were no significant differences in age or gender between the groups. Eight patients in the congenital CMV infection group were diagnosed as having autism spectrum disorder, and 15 patients had deafness. The frequencies of autism spectrum disorder and deafness were significantly higher in the congenital CMV infection group than in controls.

The median hippocampal diameter ratio was 0.99 (range: $0.70-1.58$ ) on the right side and 0.85 (range: $0.66-1.39$ ) on the left side in the congenital CMV infection group, with respective values of 0.71 (range: $0.58-0.91$ ) and 0.70 (range: $0.50-1.00$ ) in the control group. The hippocampal diameter ratio was significantly higher in the congenital CMV infection group than in controls for both sides (-Fig. 2). Defined as a hippocampal diameter ratio of $>0.92$, HIMAL was found in 17 of 34 hippocampi (50\%) in the congenital CMV infection group and 1 of 34 hippocampi (2.9\%) in controls which was a significant difference $(p<0.001)$. The number of patients with at least unilateral HIMAL in the congenital CMV infection group (11 of 17 cases; 64.7\%) was also significantly higher than that in controls (1 of 17 cases; 5.9\%; $p<0.001$; - Table 2). The medial distance ratio did not differ remarkably between the congenital CMV infection group and the control group (-Table 3 ). The intraclass correlation coefficients of the hippocampal diameter ratio and medial distance ratio were 0.87 and 0.84 , respectively, which indicated good reliability of the data.

The mean \pm standard deviation IQ or DQ of patients with congenital CMV infection was $75.8 \pm 26.1$. No remarkable correlations were seen between IQ or DQ and hippocampal diameter ratio for either side. Eight patients had autism spectrum disorder in the congenital CMV infection group. The left-side hippocampal diameter ratio tended to be higher in patients with autism spectrum disorder $(1.05 \pm 0.24)$ than in patients without $(0.85 \pm 0.22)$, albeit not significantly $(p=0.098)$. Only one patient with congenital CMV infection had epilepsy. She also displayed bilateral HIMAL and broad cortical dysplasia with microcephaly. Her seizures were observed from the age of 1 year, with focal motor seizures originating from both the right and left frontal lobes.

\section{Discussion}

Central nervous system complications in congenital CMV infection exhibit a wide spectrum of afflictions, from severe cerebral cortical dysplasia such as agyria and lissencephaly to minor white matter lesions without cortical abnormalities. Although hippocampal involvement, including abnormal formation and atrophy, has been reported in cases of diffuse cerebral cortical dysplasia, ${ }^{18}$ there are no investigations focused on hippocampal lesions in patients with congenital CMV infection to date. In the present study, most cases did not display cerebral cortical dysplasia, whereas white matter lesions were present (-Table 1). HIMAL was frequently observed in congenital CMV infection (64.7\% of patients and $50 \%$ of total hippocampi) in spite of no diffuse abnormal cerebral structures. Thus, hippocampal formation may likely be impaired, even if cerebral cortical formation is not, in patients with congenital CMV infection. The hippocampal diameter ratios in our results were widely distributed. Possible reasons for this were that HIMAL was also 

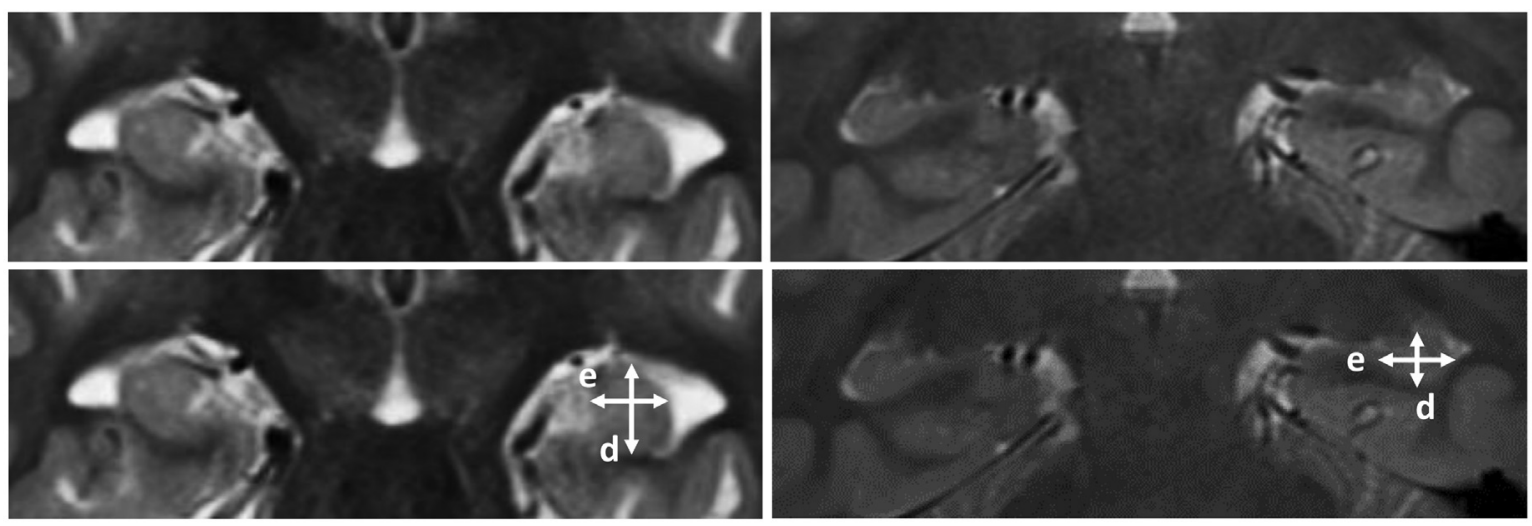

A

HIMAL (left)

Normal
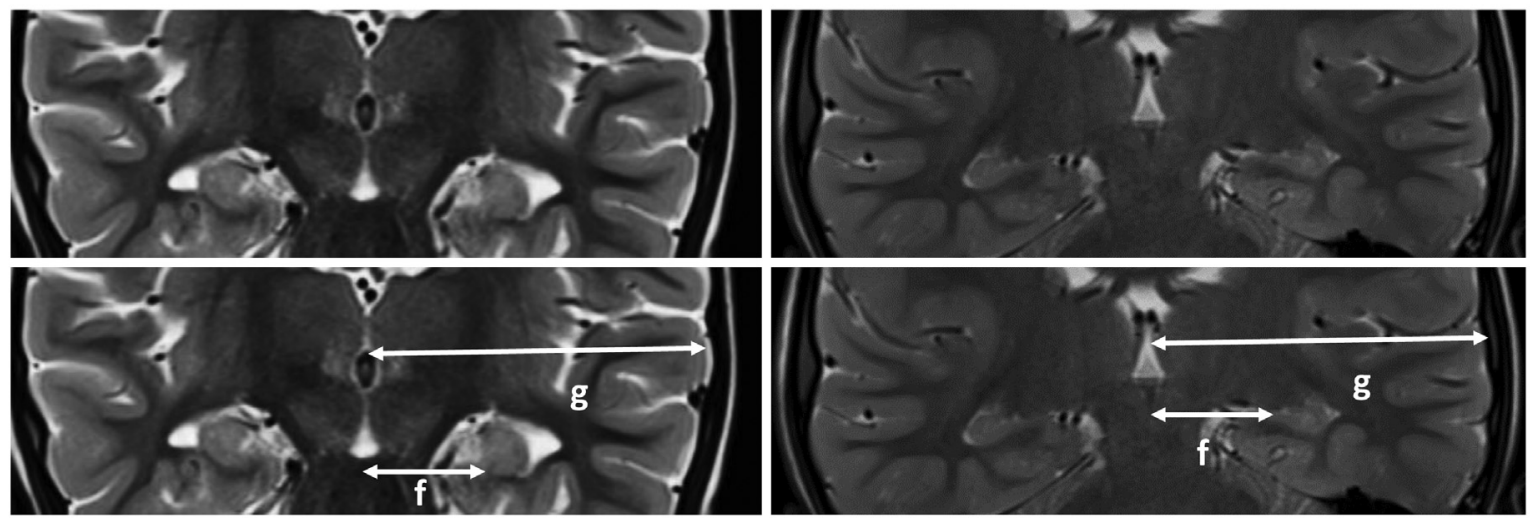

B

\section{HIMAL (left)}

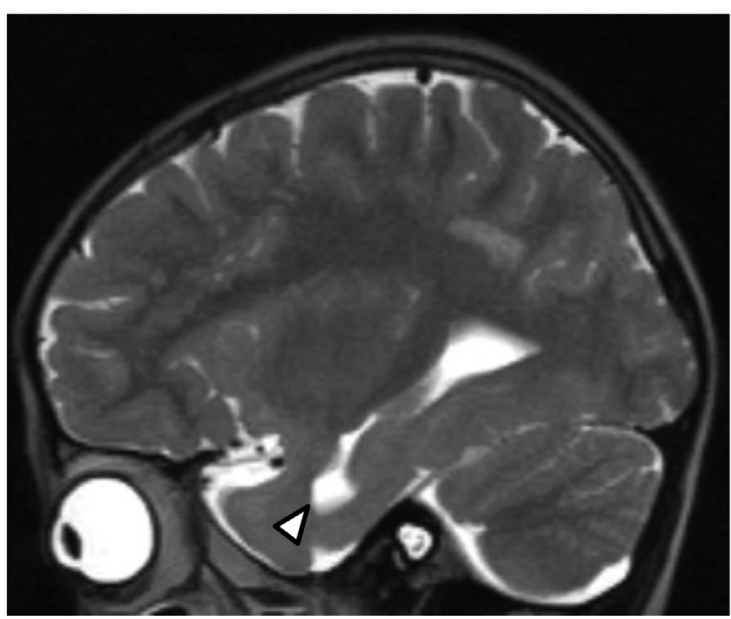

C
HIMAL

\section{Normal}

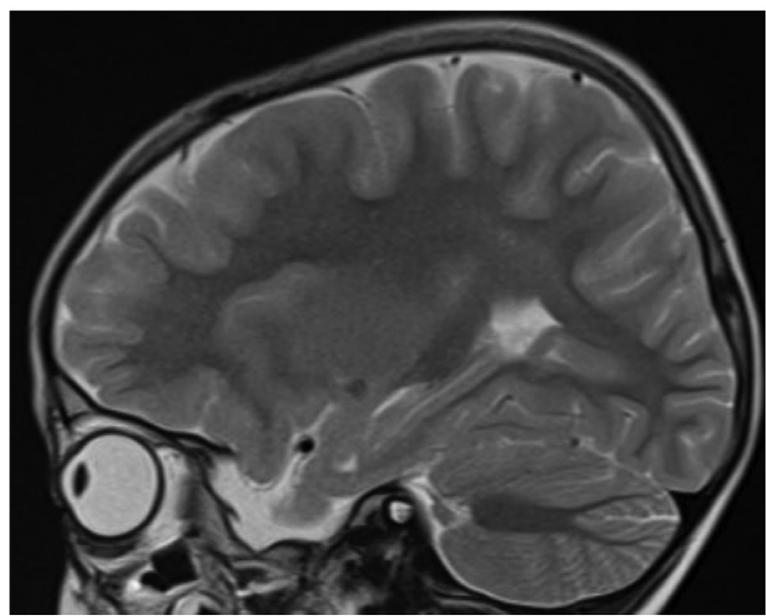

Normal

Fig. 1 Representative figures of HIMAL and the normal hippocampus. (A) Measurement of hippocampal diameter ratio (HDR). HDR =(d, height of hippocampus) / (e, width of hippocampus). (B) Measurement of medial distance ratio (MDR). MDR $=$ (f, distance from midline to medial hippocampus) / (g, distance from midline to temporal neocortex). Magnetic resonance image sections for measurement were at the slice perpendicular to the hippocampal axis, where the width of the hippocampus was maximal in the hippocampal body. (C) Sagittal images at the slice of the inferior horn of the lateral ventricle in HIMAL. Enlargement of the inferior horn of the lateral ventricle is evident (arrow head). HIMAL, hippocampal malrotation. 
observed in healthy patients, ${ }^{15}$ and that the severity of the lesion injured by CMV infection contributed to a wide ratio distribution in the congenital CMV infection group. To avoid a fallacious false, we validated our findings using Welch's $t$ test to evaluate the significance of differences in hippocampal diameter ratio in both groups and the Chi-square test to assess the occurrence rate of HIMAL between the groups.

Tsai et al revealed the features most correlated with an expert diagnosis of HIMAL to be hippocampal shape change with hippocampal diameter ratio of $>0.8$, a lack of normal lateral convex margin, and a deep dominant inferior temporal sulcus (DITS) with a DITS height ratio of $>0.6 .{ }^{15}$ Among them, the hippocampal diameter ratio is an indicator of hippocampal shape change (rounded/pyramidal) and is essential for the identification of HIMAL. Accordingly, we adopted this parameter as a simple measure of HIMAL. As this study was retrospective, T2-weighted coronal crosssectional images perpendicular to the AC-PC line according to routine protocols were available for analysis. This image angle was oblique to that of Tsai's methods which were perpendicular to the hippocampal long axis. Therefore, hippocampal angle was assumed as 30degrees based on a previous study, ${ }^{17}$ and the cut-off value of the hippocampal diameter ratio was corrected from 0.8 to 0.92 . When the hippocampal diameter ratio was above this cut-off value, hippocampal shape change was obvious, and so we were able to evaluate HIMAL without any significant differences among the examiners. Taken together, a hippocampal diameter ratio of $>0.92$ might be a good indicator for HIMAL in coronal sections of MRI perpendicular to the AC-PC line.

In the fetal brain, CMV has an affinity for periventricular embryonic cells. As these cells migrate toward the cortex to form the cerebral cortex, CMV infection impairs embryonic cells and causes various degrees of cortical dysplasia. Infections before the 18th week of gestation evoke lissencephaly and cerebellar hypoplasia, while those from 18th to 24th week result in gyrus anomalies such as polymicrogyria. ${ }^{18,19}$ Infection after the 24th week causes white matter lesions due to myelination failure which leads to impaired oligodendrocytes. ${ }^{20}$ Since hippocampal rotation is completed by the 25th week of gestation, fetal hippocampal formation is similar to that of an adult after gestation week $25 .{ }^{12}$ Hence, it is presumed that in patients with HIMAL in the absence of cortical dysplasia, CMV may have invaded the tissue between the 18th and 25th weeks of gestational age.

Regarding the pathological significance of HIMAL, this phenomenon is well recognized in epilepsy patients, especially those with medial temporal lobe epilepsy, as HIMAL is considered as an epileptogenic lesion. ${ }^{13}$ However, HIMAL and the suspected epileptogenic side are not necessarily ipsilateral, ${ }^{15}$ and there is no conclusive evidence that HIMAL is a precursor lesion progressing to hippocampal sclerosis. ${ }^{21,22}$ Moreover, HIMAL is a normal anatomical variant found in approximately $20 \%$ of healthy adults. ${ }^{15}$ On the other hand, Raininko et al described that HIMAL was not an etiologic factor in epilepsy but could be a sign of disturbed cerebral development affecting other parts of the brain, thus leading to epilepsy. ${ }^{23}$ Andrade et al reported HIMAL in $64 \%$ of patients harboring 22q11.2 deletion syndrome with or without epilepsy. ${ }^{24}$ In this study, only one patient (5.9\%) had epilepsy and HIMAL despite 11 of 17 (64.7\%) CMV infection cases with HIMAL. Our epilepsy and HIMAL case also had cortical dysplasia. Elsewhere, the epilepsy complication rate in congenital CMV infection is estimated at $26 \% .^{6}$ Based on the above-mentioned results, HIMAL may not necessarily predispose patients with congenital CMV infection to
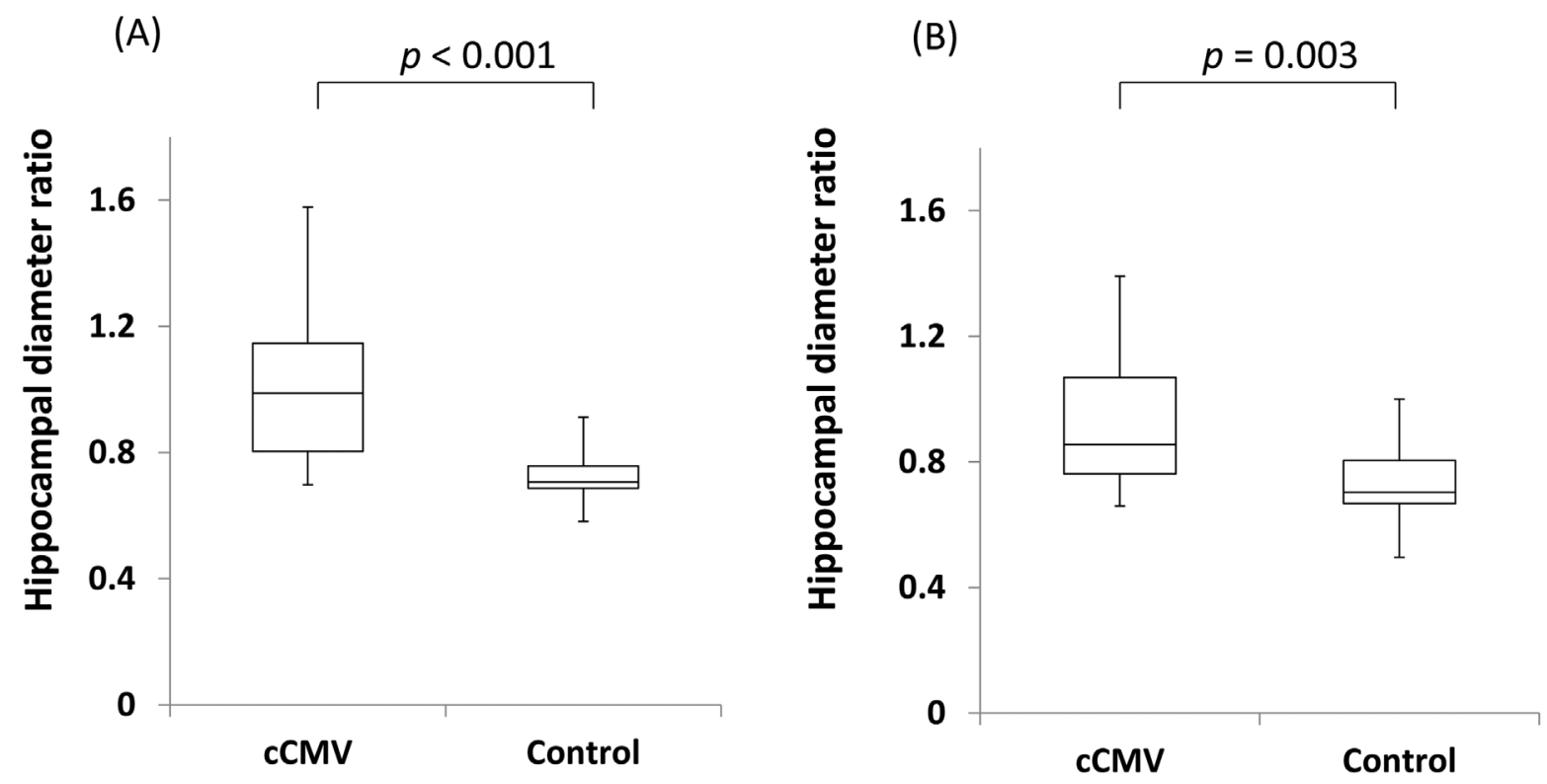

Fig. 2 Comparisons of hippocampal diameter ratio between the congenital CMV infection group and control group. There were significant differences for both the $(A)$ right side $(p<0.001)$ and $(B)$ left side $(p=0.003)$. Box whisker plots show 3horizontal bars indicating the 75 th percentile, median, and 25 th percentile values, respectively. Vertical bars indicate the mean maximal and minimal values. cCMV, congenital cytomegalovirus infection. 
Table 2 Occurrence of hippocampal diameter ratio $>0.92$

\begin{tabular}{|l|l|l|l|}
\hline & $\begin{array}{l}\text { cCMV } \\
\text { group }\end{array}$ & $\begin{array}{l}\text { Control } \\
\text { group }\end{array}$ & $p$-Value \\
\hline Number & 17 & 17 & \\
\hline Right & 4 & 0 & \\
\hline Left & 1 & 1 & \\
\hline Bilateral & 6 & 0 & \\
\hline Total hippocampi & $17(50.0 \%)$ & $1(2.9 \%)$ & $<0.001$ \\
\hline Total patients & $11(64.7 \%)$ & $1(5.9 \%)$ & $<0.001$ \\
\hline
\end{tabular}

Abbreviation: $\mathrm{CCMV}$, congenital cytomegalovirus infection.

Table 3 Comparison of medial distance ratio between the cCMV group and control group

\begin{tabular}{|l|l|l|l|}
\hline & cCMV group & Control group & $p$-Value \\
\hline Number & 17 & 17 & \\
\hline Right & $0.393 \pm 0.033$ & $0.376 \pm 0.038$ & 0.171 \\
\hline Left & $0.353 \pm 0.043$ & $0.351 \pm 0.041$ & 0.889 \\
\hline
\end{tabular}

Abbreviation: cCMV, congenital cytomegalovirus infection.

epileptogenesis. It has been reported that HIMAL more commonly affects the left-side hippocampus in both unselected epilepsy patients and controls. ${ }^{14,15,25,26}$ A multicenter investigation of over 2,000 healthy patients detected HIMAL in 17 and 6\% of left and right hippocampi, respectively. ${ }^{27}$ It is said that left predominance relates to evidence of faster right-sided hippocampal development. ${ }^{28}$ In our study, although HIMAL was more frequently observed on the left side in the control group, it was predominant on the right side in the congenital CMV infection group.

Lastly, Stiers et al suggested that HIMAL had no direct memory repercussions but rather was secondary to subtle, but widespread neurologic abnormalities also affected the morphology and function of the prefrontal cortex. ${ }^{29}$ Schizophrenia was also reportedly related to HIMAL. ${ }^{30}$ This notion was consistent with patients having congenital CMV infection in addition to displaying characteristics of autism spectrum disorder. Although the present study showed no significant correlations between HIMAL and autism spectrum disorder or IQ/DQ, the left-side hippocampal diameter ratio tended to be higher for autism spectrum disorder than that of the right side. As previous studies have shown an association between white matter lesions and IQ, the effects of HIMAL on neural function require further study.

\section{Limitations}

This study had several limitations. First, quantitative analysis of the hippocampus is necessary for evaluating HIMAL by means of coronal cross-sectional images perpendicular to the hippocampal long axis obtained by three-dimensional T1-weighted images or high-resolution T1-weighted MRI. However, as this study was retrospective, only T2-weighted coronal cross-sectional images perpendicular to the AC-PC line according to routine protocols were available for analysis. Although we corrected the cut-off value based on the hippocampal angle, angles varied among individuals. In addition, not only the hippocampal angle, but also the difference in slice thicknesses in MRI, might have influenced the height of the hippocampus. Future investigations will require coronal cross-sectional images perpendicular to the hippocampal long axis using three-dimensional T1-weighted images or high-resolution T1-weighted MRI. Second, this investigation could not address the association between hippocampal diameter ratio and age. Third, we did not detect correlations between HIMAL and epilepsy or developmental disorders, although we presumed this to be due to the limited cohort size. Future larger studies will also include quantitative evaluations of autistic behaviors to detect correlations with hippocampal diameter ratio.

\section{Conclusion}

In conclusion, this is the first report demonstrating the occurrence of HIMAL, defined here as a hippocampal diameter ratio of $>0.92$ to be significantly higher in patients with congenital CMV infection than in age-matched controls. No statistical correlations between HIMAL and IQ/DQ or the occurrence of autism spectrum disorder or epilepsy were detected, all of which warrant confirmation in larger future studies.

\section{Funding}

This work was supported by JSPS KAKENHI (grant number: JP16K09986).

Conflict of Interest

None declared.

\section{References}

1 Boppana SB, Pass RF, Britt WJ, Stagno S, Alford CA. Symptomatic congenital cytomegalovirus infection: neonatal morbidity and mortality. Pediatr Infect Dis J 1992;11(02):93-99

2 Ivarsson SA, Lernmark B, Svanberg L. Ten-year clinical, developmental, and intellectual follow-up of children with congenital cytomegalovirus infection without neurologic symptoms at one year of age. Pediatrics 1997;99(06):800-803

3 Pass RF, Stagno S, Myers GJ, Alford CA. Outcome of symptomatic congenital cytomegalovirus infection: results of long-term longitudinal follow-up. Pediatrics 1980;66(05):758-762

4 Zhang XW, Li F, Yu XW, Shi XW, Shi J, Zhang JP. Physical and intellectual development in children with asymptomatic congenital cytomegalovirus infection: a longitudinal cohort study in Qinba mountain area, China. J Clin Virol 2007;40(03): 180-185

5 Barbi M, Binda S, Caroppo S, Primache V. Neonatal screening for congenital cytomegalovirus infection and hearing loss. J Clin Virol 2006;35(02):206-209

6 Uematsu M, Haginoya K, Kikuchi A, et al. Asymptomatic congenital cytomegalovirus infection with neurological sequelae: a retrospective study using umbilical cord. Brain Dev 2016;38(09): 819-826

7 Boppana SB, Fowler KB, Vaid Y, et al. Neuroradiographic findings in the newborn period and long-term outcome in children with 
symptomatic congenital cytomegalovirus infection. Pediatrics 1997;99(03):409-414

8 Inaba Y, Motobayashi M, Nishioka M, et al. Correlation between white matter lesions and intelligence quotient in patients with congenital cytomegalovirus infection. Pediatr Neurol 2016; 55:52-57

9 Barsi P, Kenéz J, Solymosi D, et al. Hippocampal malrotation with normal corpus callosum: a new entity? Neuroradiology 2000;42 (05):339-345

10 Baulac M, De Grissac N, Hasboun D, et al. Hippocampal developmental changes in patients with partial epilepsy: magnetic resonance imaging and clinical aspects. Ann Neurol 1998;44(02):223-233

11 Bernasconi N, Kinay D, Andermann F, Antel S, Bernasconi A. Analysis of shape and positioning of the hippocampal formation: an MRI study in patients with partial epilepsy and healthy controls. Brain 2005;128(pt. 10):2442-2452

12 Bajic D, Ewald U, Raininko R. Hippocampal development at gestation weeks 23 to 36 . An ultrasound study on preterm neonates. Neuroradiology 2010;52(06):489-494

13 Matsufuji M, Utsunomiya H, Inoue T, Yasumoto S, Takashima S, Mitsudome A. Magnetic resonance imaging volumetry and clinical analysis of epilepsy patients with unilateral hippocampal abnormality. Pediatr Int 2012;54(01):19-26

14 Gamss RP, Slasky SE, Bello JA, Miller TS, Shinnar S. Prevalence of hippocampal malrotation in a population without seizures. AJNR Am J Neuroradiol 2009;30(08):1571-1573

15 Tsai MH, Vaughan DN, Perchyonok Y, et al. Hippocampal malrotation is an anatomic variant and has no clinical significance in MRI-negative temporal lobe epilepsy. Epilepsia 2016;57(10): 1719-1728

16 Furutate S, Iwasaki S, Nishio SY, Moteki H, Usami S. Clinical profile of hearing loss in children with congenital cytomegalovirus (CMV) infection: CMV DNA diagnosis using preserved umbilical cord. Acta Otolaryngol 2011;131(09):976-982

17 Gao FQ Black SE, Leibovitch FS, Callen DJ, Lobaugh NJ, Szalai JP. A reliable MR measurement of medial temporal lobe width from the Sunnybrook Dementia Study. Neurobiol Aging 2003;24(01):49-56

18 Barkovich AJ, Lindan CE. Congenital cytomegalovirus infection of the brain: imaging analysis and embryologic considerations. AJNR Am J Neuroradiol 1994;15(04):703-715

19 Bosnjak VM, Daković I, Duranović V, Lujić L, Krakar G, Marn B. Malformations of cortical development in children with congeni- tal cytomegalovirus infection - A study of nine children with proven congenital cytomegalovirus infection. Coll Antropol 2011; 35(Suppl 1):229-234

20 van der Voorn JP, Pouwels PJ, Vermeulen RJ, Barkhof F, van der Knaap MS. Quantitative MR imaging and spectroscopy in congenital cytomegalovirus infection and periventricular leukomalacia suggests a comparable neuropathological substrate of the cerebral white matter lesions. Neuropediatrics 2009;40(04): 168-173

21 Sen A, Thom M, Martinian L, Dawodu S, Sisodiya SM. Hippocampal malformations do not necessarily evolve into hippocampal sclerosis. Epilepsia 2005;46(06):939-943

22 Tsai MH, Pardoe HR, Perchyonok Y, et al. Etiology of hippocampal sclerosis: evidence for a predisposing familial morphologic anomaly. Neurology 2013;81(02):144-149

23 Raininko R, Bajic D. "Hippocampal malrotation": no real malrotation and not rare. AJNR Am J Neuroradiol 2010;31(04):E39 PubMed

24 Andrade DM, Krings T, Chow EW, Kiehl TR, Bassett AS. Hippocampal malrotation is associated with chromosome 22q11.2 microdeletion. Can J Neurol Sci 2013;40(05):652-656

25 Bajic D, Kumlien E, Mattsson P, Lundberg S, Wang C, Raininko R. Incomplete hippocampal inversion-is there a relation to epilepsy? Eur Radiol 2009;19(10):2544-2550

26 Bajic D, Wang C, Kumlien E, et al. Incomplete inversion of the hippocampus-a common developmental anomaly. Eur Radiol 2008;18(01):138-142

27 Cury C, Toro R, Cohen F, et al; IMAGEN Consortium. Incomplete hippocampal inversion: a comprehensive MRI Study of over 2000 subjects. Front Neuroanat 2015;9:160

28 Bajic D, Canto Moreira N, Wikström J, Raininko R. Asymmetric development of the hippocampal region is common: a fetal MR imaging study. AJNR Am J Neuroradiol 2012;33(03): 513-518

29 Stiers P, Fonteyne A, Wouters H, D’Agostino E, Sunaert S, Lagae L. Hippocampal malrotation in pediatric patients with epilepsy associated with complex prefrontal dysfunction. Epilepsia 2010;51(04):546-555

30 Connor SE, Ng V, McDonald C, et al. A study of hippocampal shape anomaly in schizophrenia and in families multiply affected by schizophrenia or bipolar disorder. Neuroradiology 2004;46(07): 523-534 